\title{
The HADES Tracking System
}

\author{
C. Müntz ${ }^{*, e}$, J. Markert $^{\mathrm{e}}$, G. Agakichiev ${ }^{\mathrm{c}}, \mathrm{H} \cdot$ Alvarez-Pol $^{\mathrm{f}}$, \\ E. Badura ${ }^{\text {a }}$, J. Bielcik ${ }^{\text {a }}$, H. Bokemeyer ${ }^{\text {a }}$, J.-L. Boyard ${ }^{\text {b }}$, \\ V. Chepurnov ${ }^{c}$, S. Chernenko ${ }^{c}$, H. Daues ${ }^{a}$, F. Dohrmann ${ }^{d}$, \\ W. Enghardt ${ }^{\mathrm{d}}$, O. Fateev ${ }^{\mathrm{c}}$, C. Garabatos ${ }^{\mathrm{a}}$, L. Glonti $^{\mathrm{c}}$, \\ E. Grosse ${ }^{\mathrm{d}}$, J. Hehner ${ }^{\mathrm{a}}$, K. Heidel $^{\mathrm{d}}$, T. Hennino ${ }^{\mathrm{b}}$, \\ J. Hoffmann $^{\text {a }}$, A. Ierusalimov ${ }^{c}$, B. Kämpfer ${ }^{d}$, K. Kanaki ${ }^{d}$, \\ W. Koenig ${ }^{\text {a }}$, R. Kotte ${ }^{\mathrm{d}}$, L. Naumann ${ }^{\mathrm{d}}$, W. Ott ${ }^{\mathrm{a}}$, \\ Y.C. Pachmayer ${ }^{\mathrm{e}}$, V. Pechenov ${ }^{\mathrm{c}}$, J. Pouthas ${ }^{\mathrm{b}}$, B. Ramstein ${ }^{\mathrm{b}}$, \\ K. Rosenkranz ${ }^{\text {e }}$, P. Rosier ${ }^{\text {b }}$, M. Roy-Stephan ${ }^{\text {b }}$, A. Rustamov ${ }^{\text {a }}$, \\ A. Sadovski ${ }^{\text {d }}$, L. Smykov ${ }^{c}$, M. Sobiella ${ }^{d}$, H. Stelzer ${ }^{\mathrm{a}}$, \\ H. Stroebele ${ }^{\mathrm{e}}$,J. Stroth ${ }^{\mathrm{a}}$, C. Sturm ${ }^{\mathrm{a}}$, M. Sudol ${ }^{\mathrm{a}}$, \\ J. Wüstenfeld ${ }^{\mathrm{e}}$, Y. Zanevsky ${ }^{\mathrm{c}}$, P. Zumbruch ${ }^{\mathrm{a}}$ \\ ${ }^{a}$ Gesellschaft für Schwerionenforschung, 64291 Darmstadt, Germany \\ b IPN, 91406 Orsay, France \\ ${ }^{\mathrm{c}}$ Joint Institut of Nuclear Research, 141980 Dubna, Russia \\ ${ }^{\mathrm{d}}$ IKH, Forschungszentrum Rossendorf, 01314 Dresden, Germany \\ e Johann Wolfgang Goethe-Universität, 60486 Frankfurt, Germany \\ ${ }^{\mathrm{f}}$ Universidade de Santiago de Compostela, 15706 Santiago de Compostela, Spain \\ *corresponding author, email: c.muentz@gsi.de
}

\begin{abstract}
The HADES dielectron spectrometer has recently launched its physics program at the heavy ion synchrotron SIS at GSI Darmstadt. The spectroscopy of vector mesons in heavy ion collisions via their dielectron decay channel makes great demands on the HADES tracking system regarding acceptance and spatial resolution. The tracking system is formed out of 24 low-mass, trapezoidal multi-layer drift chambers providing about $30 \mathrm{~m}^{2}$ of active area. Low multiple scattering in the in total four planes of drift chambers before and after the magnetic field is ensured by using helium-based gas mixtures and aluminum cathode and field wires. First in-beam performance results are contrasted with expectations from simulations. Emphasis is placed on the energy loss information, exploring its relevance regarding track recognition.
\end{abstract}




\section{Introduction}

The HADES dielectron spectrometer [1] at the heavy ion synchrotron SIS at GSI Darmstadt has taken up its physics program in several beam time campaigns, starting late 2001, focusing on $\mathrm{C}+\mathrm{C}$ reactions at 1 and $2 \mathrm{AGeV}$ incident energy. Recently, a beam time studying $\mathrm{p}+\mathrm{p}$ reactions in the same energy regime has been successfully conducted.

The spectroscopy of vector mesons in heavy ion collisions via their dielectron decay channel defines the decisive design and performance constraints on the HADES tracking system. An intrinsic spatial cell resolution of better than $140 \mu \mathrm{m}$ along with the reduction of multiple scattering in detector materials and gas, high efficiency and a large acceptance are crucial requirements for the success of the experimental program. Extended design studies and prototyping $[2,3]$ preceded the production of the 24 drift chambers of four different sizes, conducted by GSI Darmstadt, LHE/JINR Dubna, FZ Rossendorf [4], IPN Orsay and University of Frankfurt.

Presently 22 chambers are in-place and do already now allow for high-precision tracking in two-third of the full azimuth. In the following the tracking system and its design parameters are introduced. Then, first results are briefly reviewed, highlighting data on the capability of the drift chamber energy loss signal regarding track matching, and particle identification in general.

\section{The HADES drift chambers}

\subsection{Detector characteristics}

The HADES tracking system consists of 24 trapezoidal, planar multi-layer drift chambers (MDC) symmetrically arranged in six identical sectors. It provides a polar angles coverage between 18 and 85 degrees around the beam axis, forming four tracking planes (I-IV) of increasing size. In each sector, two chambers (plane I and II) are located in front of and two (plane III and IV) behind the toroidal magnetic field of the super-conducting magnet, as shown in figure 1. To cope with ambiguities in track reconstruction in the high multiplicity environment of a heavy ion reaction (for central $\mathrm{Au}+\mathrm{Au}$ collisions at $1 \mathrm{AGeV}$ incident energy a maximum cell occupancy of $30 \%$ is estimated), all chambers are composed of six sense/field wire layers oriented in five different stereo angles. This allows for maximum spatial resolution in polar direction, which points in the direction of the momentum kick. All four chamber types contain about 1100 drift cells each, with increasing size from $5 \times 5 \mathrm{~mm}^{2}$ (plane I) to $14 \times 10 \mathrm{~mm}^{2}$ (plane IV). The chambers provide active areas from $0.35 \mathrm{~m}^{2}$ up to $3.2 \mathrm{~m}^{2}$, thus covering the same solid angle per sector, respectively. The main feature of the design and the operation parameters of the chambers is the consequent implementation of the low-mass concept, as result of the $R \& D$ phase [2]. To meet these requirements (i) cathode and field wires are made of annealed aluminum (plane I-III: bare, IV: gold-plated) with $80 \mu \mathrm{m}$ and 
$100 \mu \mathrm{m}$ diameter, and with initial tensions between 80 and $150 \mathrm{cN}$, depending on the chamber type. In addition, (ii) helium-based counting gas (helium:isobutane $=$ 60:40) is used. The entrance windows are made of $12 \mu \mathrm{m}$ aluminized mylar. The $20 \mu \mathrm{m}$ (planes I-III) and $30 \mu \mathrm{m}$ (plane IV) thick gold-plated tungsten sense wires are strained with an initial tension of 40 and $50 \mathrm{cN}$, respectively. To compensate for the total wire tension after being released from the assembly table, all chamber frames have been pre-stressed before wire gluing. Together with the requirements concerning the acceptance this resulted in a sophisticated layer frame design with only $3 \mathrm{~cm}$ width for the inner-most chambers. The total detector thickness per chamber in units of radiation length is below $5 \times 10^{-4}$ and hence low multiple scattering guarantees the momentum resolution needed to accomplish the physics demands.

\subsection{Long-term stability}

The experiments with HADES are expected to run at least ten years. Creeping of the aluminum wires and ageing are the main concerns with respect to the longterm stability of the chambers. Creeping has been systematically investigated in tension loss test series, yielding a $10 \%$ loss in tension within five years. This has been confirmed by remeasurements in one chamber of plane III [4]. Ageing is mainly caused by the accumulated dose in combination with the materials used for construction and operating the chambers. For example, epoxy from Araldite is used for gluing the wires on the frames from Stesalit. The gas system is running in a re-flow mode with typically $10 \%$ fresh gas and continuous purification. The drift velocity monitors [5] provide a very sensitive control of the gas quality by measuring the drift velocity with a precision of better than $0.2 \%$. In addition, the simultaneous monitoring of the relative gains allows for conclusions on the gas contamination, e.g. due to oxygen. The expected maximum dose is in the order of $10 \mathrm{mC}$ per year and centimeter of the sense wire. An accelerated ageing test with ${ }^{55} \mathrm{Fe}$ using two prototype chambers exhibited no noticeable gain drop $(<5 \%)$ for a time period of two years of running [2].

These $R \& D$ results on creeping and ageing, together with the careful selection of materials and running conditions, suggest that the projected long-term operation of the HADES tracking system can be kept.

\subsection{Read-out electronics}

The drift chamber signals are read-out and digitized at the chamber, not extending into its active area. Hence, special emphasis was put on the integration of the modular front-end electronics, realized with analog boards (16 channels) mounted on digitization boards ( 64 or 96 channels). Four sense wires are connected by flexible printed circuits to the analog boards [8], housing ASD8-B chips [9] (8 channels, $1 \mathrm{fC}$ intrinsic noise, $30 \mathrm{~mW} / \mathrm{ch}$, adjustable threshold) for differential amplification, 
shaping and discrimination. These chips deliver logical signals with variable width, equivalent to the time the shaped signal exceeds the given threshold. The logical signals are fed to TDC chips (CMOS, 8 channels/chip, $0.5 \mathrm{~ns} / \mathrm{ch}$, common-stop, $1 \mu \mathrm{s}$ full range, making use of a ring oscillator) on the digitization boards. This semi-customized ASIC is multi-hit capable and thus allows to detect also the timeabove-threshold of each hit. Besides spike and zero suppression this chip offers the possibility of internal calibration, activated by a separate trigger type. The design of the front-end electronics was decisively influenced by minimizing the noise level on-line. In addition, the time-above-threshold information is a valuable tool to discriminate remaining noise hits off-line.

\section{$3 \quad$ Results}

\subsection{Performance}

The chambers have been running in several $\mathrm{C}+\mathrm{C}$ and $\mathrm{p}+\mathrm{p}$ experiments with moderate intensity and occupancy. Layer detection efficiencies around $98 \%$ have been deduced. The average spatial resolutions of a drift cell can be determined with data by means of a self-tracking method and is between 100 and $130 \mu \mathrm{m}$, comprising contributions from calibration accuracy and electronic noise. This is well below the design value of $140 \mu \mathrm{m}$, see figure 2 . Under clean conditions, with $2.1 \mathrm{GeV} / \mathrm{c}$ protons impinging on a prototype, using a silicon-strip tracker as reference and standard read-out electronics, intrinsic spatial resolutions around $70 \mu \mathrm{m}$ have been reached [3], similar to results given by GARFIELD simulations. Preliminary simulation studies on the expected track spatial resolution in a segment of two adjacent chambers yield values below $60 \mu \mathrm{m}$ (modules of plane I and II) in direction of the momentum kick, made possible by the multiple position measurement per segment, see figure 3 . These simulations have been confirmed by experimental results from tracking, suggesting an error in measuring the angle of a segment in the polar direction of about $0.2 \mathrm{mrad}$.

\subsection{Energy loss and tracking}

Besides the arrival time of the electrons the time-above-threshold $\Delta \mathrm{t}$ of the corresponding signal is measured for each cell hit. From the latter value the energy loss can be deduced. $\Delta \mathrm{t}$ is a measure of the number of produced primary electron clusters [7], i.e. the energy loss of a particle traversing the drift cell. It is determined by the ionization density of the particle, and the track topology in a given cell, which is characterized by the impact angle and the impact parameter relative to the sense wire. The energy loss along a track (segment) can be used to resolve ambiguities in matching track segments from the inner and outer chambers, to support the particle identification by adding complementary information, and to reject tracks 
originating from conversion and Dalitz decays with small opening angles - the main background in dielectron spectroscopy.

To investigate the resolution of the energy loss signal, effects from the topology of the track as well as of the electron drift inside a cell have to be corrected for. These topological effects can be corrected by using the local information on the orientation and position of the track segment, defined by two adjacent chambers in one sector, respectively. Here, GARFIELD simulations on time-to-distance relations in different cell geometries are employed. Simulation results and measured data coincide to a high degree [7]. Effects of the electron drift topology on the energy loss signal have been systematically parameterized based on data only, and are used for normalization. As a result, the normalized $\Delta \mathrm{t}$ can be plotted as a function of the measured momentum, shown in figure 4. Clearly, the separation of pions and protons at low momenta can be seen. Electrons cannot be distinguished from pions. The achieved resolution $(\sigma)$ of the normalized $\Delta \mathrm{t}$ so far is about $7 \%$ (protons) and $12 \%$ (pions), possible improvements are currently under investigation. However, these results provide already now confidence in the analysis power of the MDC's energy loss information, although the chamber design has originally not been optimized in this respect.

\section{Conclusions}

The low-mass HADES tracking system is operational and the performance is according to its design values, thus allowing for high-resolution dielectron spectroscopy. Currently, scenarios are under debate on the possibility to include the hit informa-

tion of the chambers in a higher level trigger. The energy loss signal measured for each track turned out to be a valuable tool to improve on resolving ambiguities in tracking and on background reduction, even though the chamber design has been optimized for minimizing the detector thickness.

\section{References}

[1] see http://www-hades.gsi.de

[2] C. Garabatos et al., Nucl. Instr. Meth. A 412 (1998) 38

[3] H. Bokemeyer et al., Nucl. Instr. Meth. A 477 (2002) 397

[4] K. Kanaki et al., Conference proceedings NSS-MIC, subm. to IEEE-TNS

[5] C. Lippmann, Diploma thesis, Univ. of Frankfurt, 2001

[6] R. Veenhof, version 7.04, http://garfield.web.cern.ch

[7] J. Markert, PhD thesis in preparation, Univ. of Frankfurt

[8] E. Badura et al., Particles and Nuclei, Letters No.1[98]-2000 73

[9] M. Newcomer, IEEE Trans. on Nucl. Sc. 40 (1993) 


\section{$5 \quad$ Figure captions}

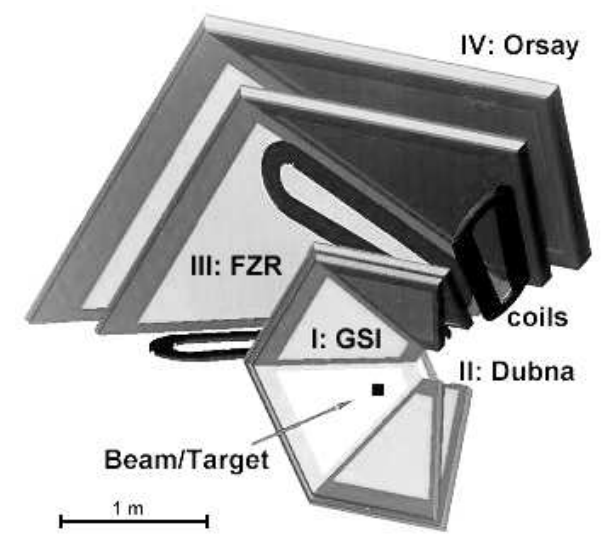

Fig. 1: Schematic view of the HADES tracking system, arranged in a frustumlike geometry before and behind the magnet coils. The four chamber construction sites are indicated.

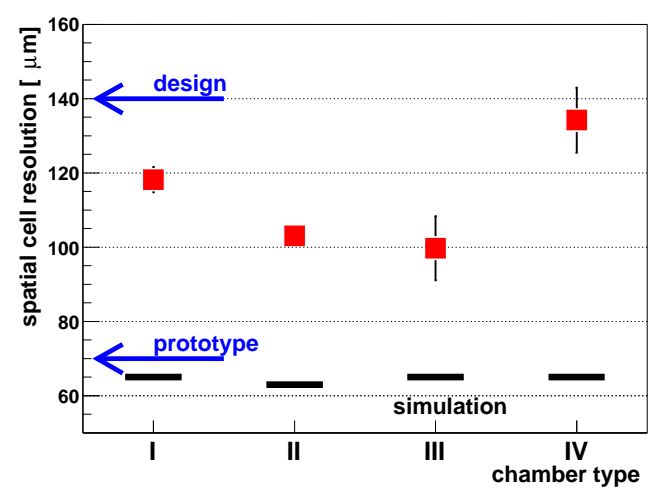

Fig. 2: Average in-beam spatial drift cell resolution (symbols), for the four chamber types I-IV present in 2001. The error bars depict the variance of several modules of one type (no systematic errors, and not corrected for contributions from read-out electronics and calibration). The data is compared to the design value, a result from prototype studies, and Garfield simulations.

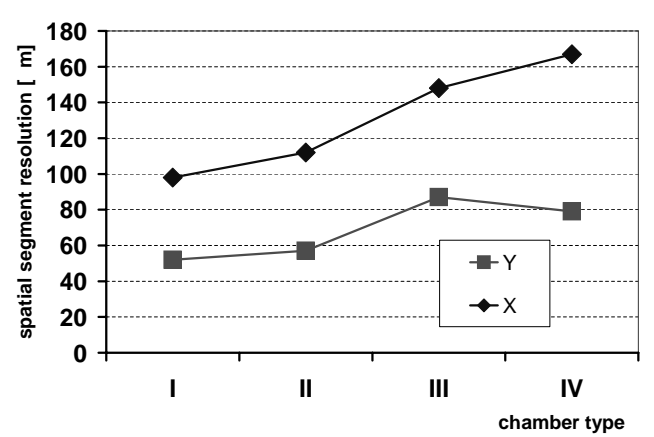

Fig. 3: Preliminary results from simulations: Spatial segment resolution for the four chamber types (I-IV), in $\mathrm{Y}$ (momentum kick direction) and perpendicular $(\mathrm{X})$.

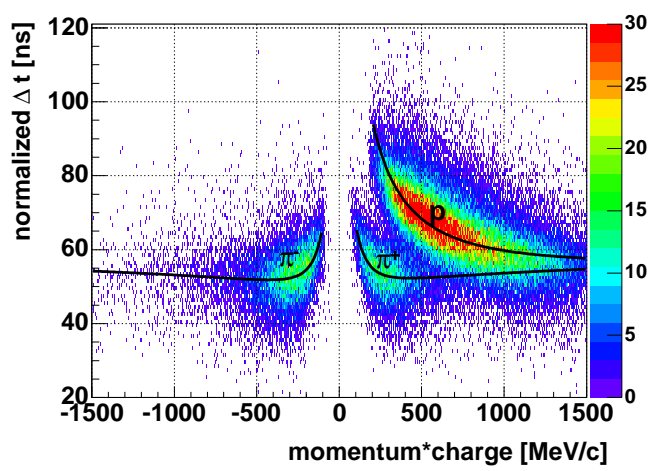

Fig. 4: Data from $\mathrm{C}+\mathrm{C}, 2 \mathrm{AGeV}$ : normalized time-above-threshold $\Delta \mathrm{t}$ is plotted as a function of the measured momentum times the charge for the inner MDC segment (planes I and II). Lines indicate Bethe-Bloch parameterizations. 